\title{
Resorption of retromolar bone grafts after alveolar ridge augmentation-volumetric changes after 12 months assessed by CBCT analysis
}

\author{
Andres Stricker ${ }^{1,2,3^{*}}$ (D), Reinhilde Jacobs ${ }^{4,5,6}$, Frederik Maes ${ }^{7}$, Tabea Fluegge ${ }^{8}$, Kirstin Vach ${ }^{9}$ and Jonathan Fleiner ${ }^{1,4}$
}

\begin{abstract}
In this pilot study, a volumetric analysis of retromolar onlay bone grafts over a period of 12 months was conducted, using repeated $\mathrm{CBCT}$ imaging combined with automated image registration.

Eleven patients being treated with 16 bone grafts taken from the retromolar area were examined by CBCT scanning prior to bone augmentation (TO), immediately after bone augmentation (T1) and after a healing time of 12 months after augmentation (T2). Graft volumes were measured at each time point after automated image registration of consecutive CBCT scans.

The mean volume of the augmented site was $372.2 \pm 179.4 \mathrm{~mm}^{3}$. Resorption relative to the original augmented volume was $43.7 \% \pm 19.0 \%$ after 12 months.

Three-dimensional graft resorption could be precisely depicted by the use of automated image registration for CBCT data over a period of 12 months and demonstrated extensive volumetric changes of bone grafts taken from the ascending ramus of the mandible.

Graft resorption and continuous bony remodeling of the grafted site before and after implant insertion have to be carefully considered by the clinician.
\end{abstract}

Keywords: Ridge augmentation, Bone remodeling, Bone resorption, Onlay graft, $\mathrm{CBCT}$, Three-dimensional imaging, Alveolar bone

\section{Introduction}

Sufficient bone quantity and quality at the recipient site is a major prerequisite for long-term success of dental implants [1-3]. However, in many situations, there is a bone deficiency, indicating a surgical procedure that predictably leads to sufficient bone quantity prior to implant insertion [4-6].

Guided bone regeneration (GBR), alveolar distraction osteogenesis, and onlay grafting have been described to

\footnotetext{
* Correspondence: andres.stricker@uniklinik-freiburg.de

'Center of Implantology, Periodontology and 3D Head-and-Neck Imaging, Konstanz, Germany

${ }^{2}$ Department of Oral and Maxillofacial Surgery, Medical Center, University of Freiburg, Freiburg, Germany

Full list of author information is available at the end of the article
}

augment the horizontal and vertical bone volume [7-10]. Transplantation of autogenous bone grafts is the standard procedure for reconstruction of a severely resorbed alveolar crest prior to implant insertion [11-19].

However, bone resorption both in height and width has been described for onlay grafts harvested from the iliac crest [20-24] and ascending ramus of the mandible [25-28].

Mechanical calipers and different radiographic techniques have been applied for assessment of the augmented areas prior to implant placement $[25,28]$. Conventional radiographic imaging, using the parallel technique, bitewings or panoramic X-rays, allows an estimation of the vertical dimension of the bone graft. However, due to the inevitable overlay of anatomical structures and the 
presence of image distortion and blurring, adequate information about the horizontal dimension and the three-dimensional volume changes cannot be derived $[29,30]$. In contrast, computed tomography (CT) and cone beam computed tomography (CBCT) technology may overcome these fundamental limitations and may be used for accurate three-dimensional representation of the alveolar bone before and after bone augmentation. Volumetric CT studies of autogenous onlay grafts harvested from the iliac crest demonstrated a rapid initial loss of bone height during the first 6 months of healing [22, 31].

Three-dimensional analysis using CBCT got increasingly popular because of its lower radiation and higher resolution compared to volumetric CT [32]. Here, longterm stability of implants inserted in the pristine bone [33-35] and assessment of onlay bone graft have been studied [36-39].

In order to enable adequate prediction of volumetric changes of autogenous onlay bone grafts over time, the aim of this pilot study was to conduct a volumetric analysis using $\mathrm{CBCT}$ imaging at different postoperative time points combined with an automated image registration procedure [40], to accurately evaluate volume alterations of onlay graft augmentation with autogenous bone taken from the ascending ramus of the mandible over a period of 12 months.

\section{Materials and methods \\ Patient selection}

In this study, patients of at least 18 years with autogenous bone grafts from the ascending alveolar ramus prior to dental implant placement were considered for this retrospective analysis. Study approval was obtained from the Ethical Committee of the University Medical Center Freiburg, Germany (138/14).

All patients were healthy, non-smokers and had no general contraindications as history of malignancy, antiresorptive-, radio- or chemotherapy, pregnancy or nursing, and general diseases which may negatively affect bone or connective tissue metabolism or bone turnover rate.

Local inclusion factors comprised a transversal width of the alveolar crest $<3 \mathrm{~mm}$ and a vertical height $>7$ $\mathrm{mm}$.

In addition, for retrospective inclusion in the study independently of study aspects, CBCT scans must have been acquired at different time points:

- Prior to bone augmentation (T0)

- Immediately after bone augmentation (T1))

- 12 months after bone augmentation (T2).

A total of 220 patients being treated with retromolar bone grafts were initially screened for inclusion in this study. Based on the criteria above, 11 patients (10 female, 1 male) having a mean age of 53.0 years (range 20-69 years) could be selected for further analysis.

\section{Surgical procedure}

Bone augmentation was performed with local anesthesia in 8 patients, while 3 patients were operated under general anesthesia.

After crestal incision and elevation of a muco-periostal flap, the atrophy of the recipient site was evaluated. Thereafter, a paramarginal incision in the retromolar region of the mandible was performed to get access for harvesting the cortico-cancellous bone graft from the ascending ramus of the mandible using a piezotome and chisel.

Bone grafts were adapted to atrophic ridge anatomy with diamond burs before fixation to the recipient bone by two titanium screws of $1.5 \mathrm{~mm}$ diameter and $8 \mathrm{~mm}$ length (Bone Fixation Aesculap, Tuttlingen, Germany). Periostal release incision was followed by tension free wound closure with non-resorbable sutures (Seralon 5.0, Serag Wiessner, Germany). After surgery, antibiotics (Clindamycin $600 \mathrm{mg}, 3 \times / \mathrm{d}, 7$ days), pain medication (Ibuprofen 400), and rinsing irrigation (0.1\% CHX rinsing solution) were administered.

\section{$\mathrm{CBCT}$ analysis}

CBCT scans acquired at different time points were evaluated to assess volumetric changes of onlay grafts over time. CBCT scanning was performed prior to bone augmentation (T0), immediately after bone augmentation (T1), and at 12 months after bone augmentation (T2).

All CBCT scans were acquired with an iCat Cone Beam 3D scanner (Imaging Sciences International, LLC, Hatfield, PA, USA) with the following scan parameters: $0.3 \mathrm{~mm}$ voxel size, $5 \mathrm{~mA}, 120 \mathrm{kV}$, and scanning time 8.9 s.

For each patient, the baseline scan (T0) was aligned to the post-op scan (T1) and the follow-up scan (T2,) using an automated image registration procedure [40]. A rigid registration was used that was restricted to either the mandible or the maxilla, depending on the implant site. The accuracy of the registration was visually verified for all cases. In this way, identic orientation of the anatomic details of the region of interest (ROI) could be guaranteed.

In a next step, the bony contours of the ROI for T0 were delineated and visualized against every aligned follow-up scan (T1, T2) to ensure bone volume measurements within identical anatomical regions at all time points (Figs. 1, 2, and 3). The additional amount of grafted bone extending the defined contours of the T0 region was measured by manual slice-by-slice delineation in the axial CBCT images (Figs. 1, 2, and 3). In vertical direction, axial CBCT slices between the marginal 


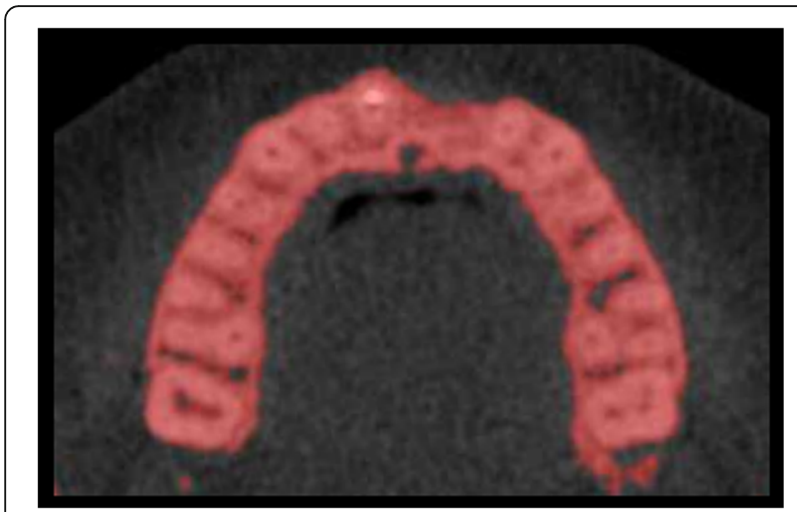

Fig. 1 CBCT scan (axial view) of lower jaw at time point T0 before onlay graft

alveolar bone crest and the level of the maximal extent of the onlay graft were considered [41]. Consequently, the total volume of the augmented site was calculated by automated interpolation between the axial slices $(\mathrm{MeVi}$ sLab V.1, MeVis Medical Solutions AG, Bremen, Germany). All CBCT scans were analyzed by two independent examiners that were not involved in the surgical therapy of these patients.

\section{Statistical evaluation}

For this study, all available patients with a CBCT scan 12 months after augmentation were used; hence, no sample size calculation was performed. For the $11 \mathrm{pa}-$ tients, an effect size of 2.3 can be detected.

For descriptive analysis, mean and standard deviation were computed. Intraclass correlation coefficients (ICC) were used to evaluate the agreement between the two observers for the different volume measurements. For further analysis, the mean volume values over the observers were used. A paired $t$ test was applied to check for differences between the original bone volume and the 12-month value. Linear regression models with robust variance estimators were used to analyze both influence of jaw and age on the volume value.
All calculations were performed with the statistical software STATA 16 (StataCorp LT, College Station, TX, USA). The probability level for statistical significance was set to $P<0.05$.

\section{Results}

Surgical rehabilitation affected 11 patients having 16 bone grafts, 4 in the maxilla, and 12 in the mandible, respectively.

All onlay bone grafts healed uneventfully, without soft tissue dehiscence, and graft incorporation was successful to allow placement of 22 dental implants after a healing time of $104.4 \pm 11.5$ days.

Final prosthodontic rehabilitation by implant-supported restorations was performed 105.2 \pm 17.7 days after implant placement or $209.6 \pm 24.6$ days after bone augmentation. No implants were lost during the entire observation period of $510.4 \pm 180.2$ days and thereafter up to now.

We observed a very high intraclass correlation between the volume measurements of the two observers for all time points of $0.999(p<0.0001)$. For further analyses, the mean volume measurements over the two observers were used (Fig. 4).

The mean bone volume of the augmented site was $372.2 \pm 179.4 \mathrm{~mm}^{3}$ immediately post-operatively (T1) and $230.2 \pm 185.7 \mathrm{~mm}^{3}$ after 12 months (T2), leading to an extent of total resorption relative to the original bone volume of $43.7 \% \pm 19.0 \%$, being highly significant $(p<$ 0.001) (Figs. 4 and 5).

Furthermore, the influence of age and jaw on the volume change was analyzed. We observed a regression coefficient of -1.73 for age, meaning that the volume change from baseline to the last time point became lower with increasing age, but was not significant $(p=0.175)$.

Localization of onlay graft augmentation demonstrated differences: a mean volume change from baseline to T2 in the lower jaw was $113.7 \pm 34.7 \mathrm{~mm}^{3}(37.6 \% \pm 16.7 \%)$. In the upper jaw, the difference was larger $217.7 \pm 56.2 \mathrm{~mm}^{3}$ $(60.2 \% \pm 16.4 \%)$, being statistically significant $(p=0.01)$.

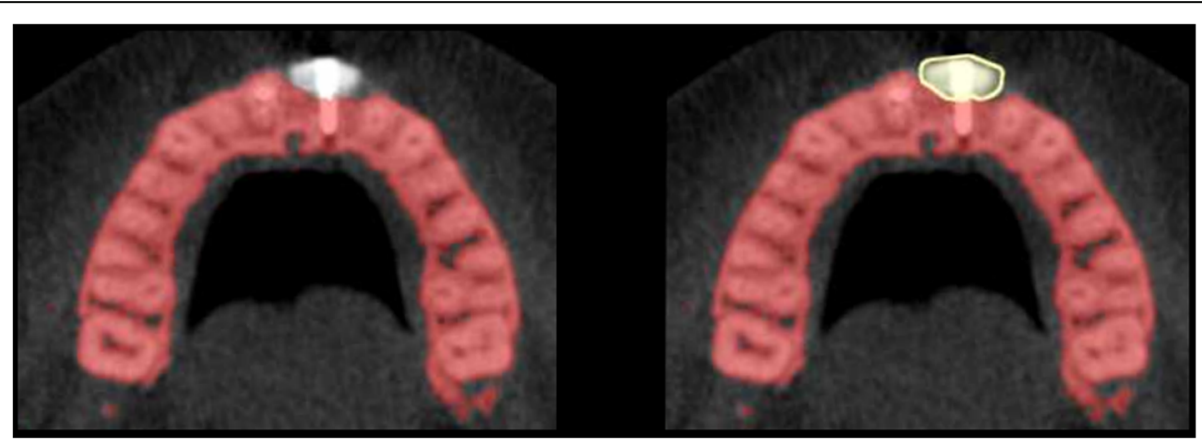

Fig. 2 CBCT scan (axial view) of lower jaw at time point T1 postoperatively after onlay graft 


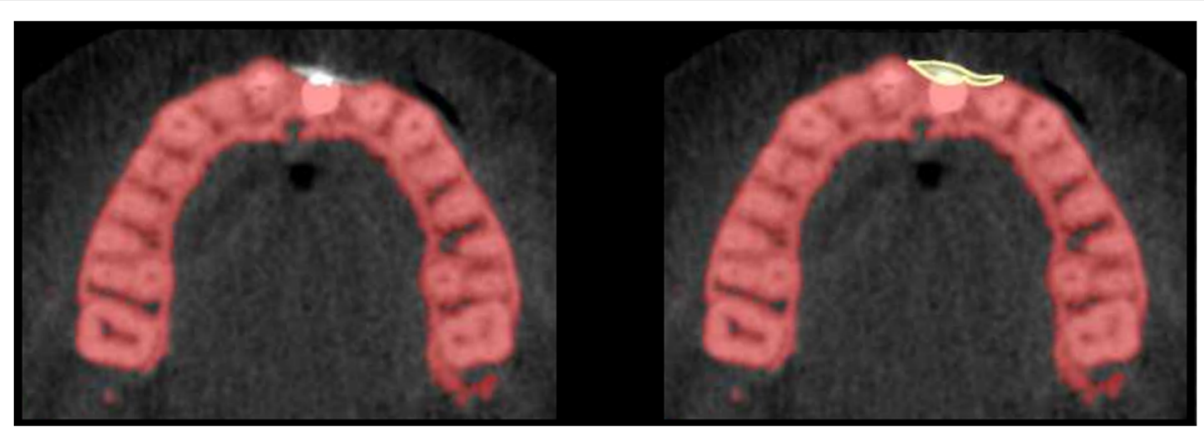

Fig. 3 CBCT scan (axial view) of lower jaw at time point T2, 12 months after onlay graft

\section{Discussion}

This pilot study is a three-dimensional CBCT study using an automated image registration procedure to analyze bone resorption of onlay grafts harvested from the ascending ramus of the mandible.

The use of cross-sectional information provided by CT scans to examine bone resorption of onlay grafts was introduced by Nyström et al. in 1995 [42]. The authors performed linear measurements of width and height of iliac onlay grafts in arbitrarily defined two-dimensional cross-sections oriented along the simultaneously inserted implants [42]. A comparable measurement protocol was followed by Malchiodi et al. to measure bone width in axial CT cross-sections of augmented maxillae and mandibles [26]. The previously described methods make use of CT technology to assess bone width in oro-vestibular direction; however, a volumetric assessment of bone grafts was not performed.

Anitua et al. and Monje et al. transferred linear measurement protocols to cross-sectional images derived from CBCT imaging and measured bone width of onlay grafts at defined levels above the residual alveolar bone [37, 39].

Volumetric measurement protocols using CT technology were mostly used to assess resorption of iliac onlay grafts [31, 43-45]. The outline of the grafted areas was manually delineated in multiple cross-sections of the complete onlay graft and added to obtain its volume. The orientation of cross-sections and the use of landmarks varied and authors did not disclose their protocol to differentiate between bone grafts and residual bone, especially after healing of bone grafts.

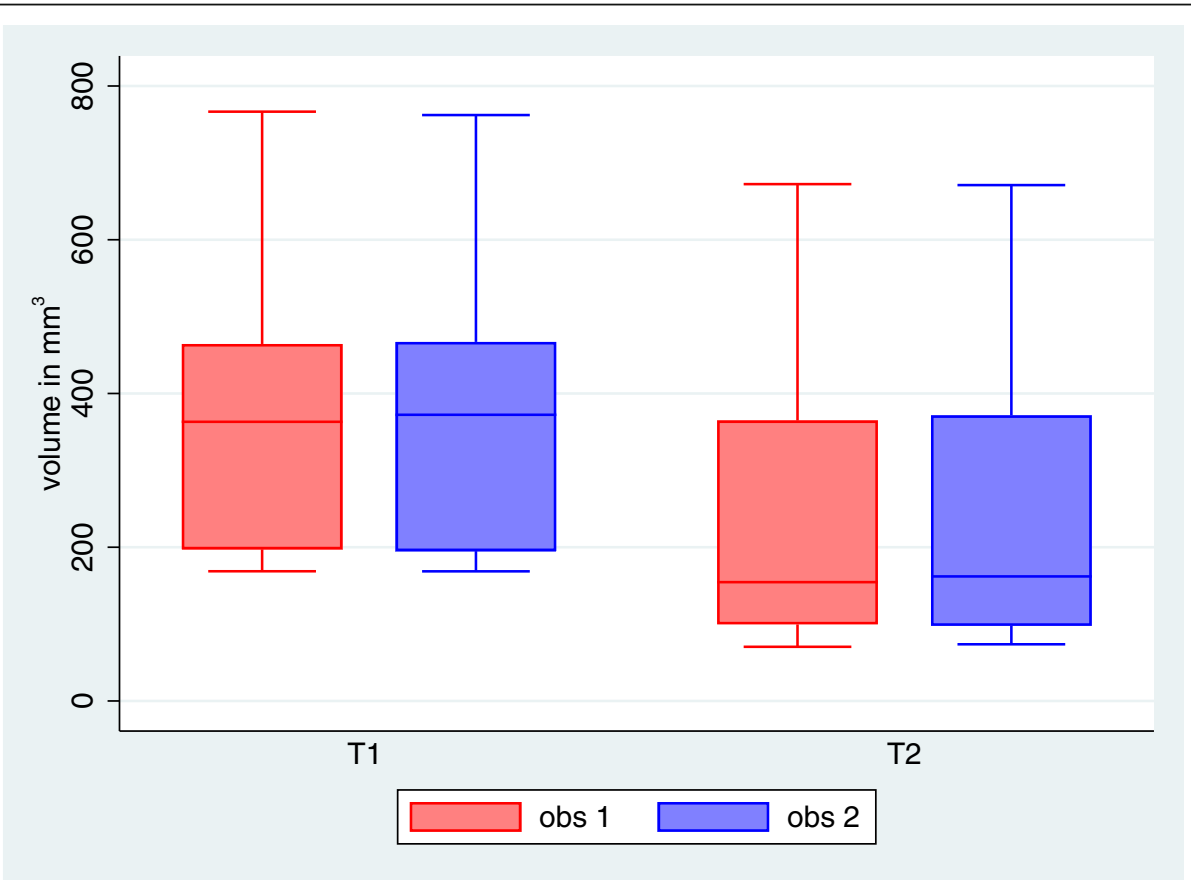

Fig. 4 Mean bone volume within identical ROls at different time points T1 and T2 after augmentation with onlay grafts harvested from the retromolar area 


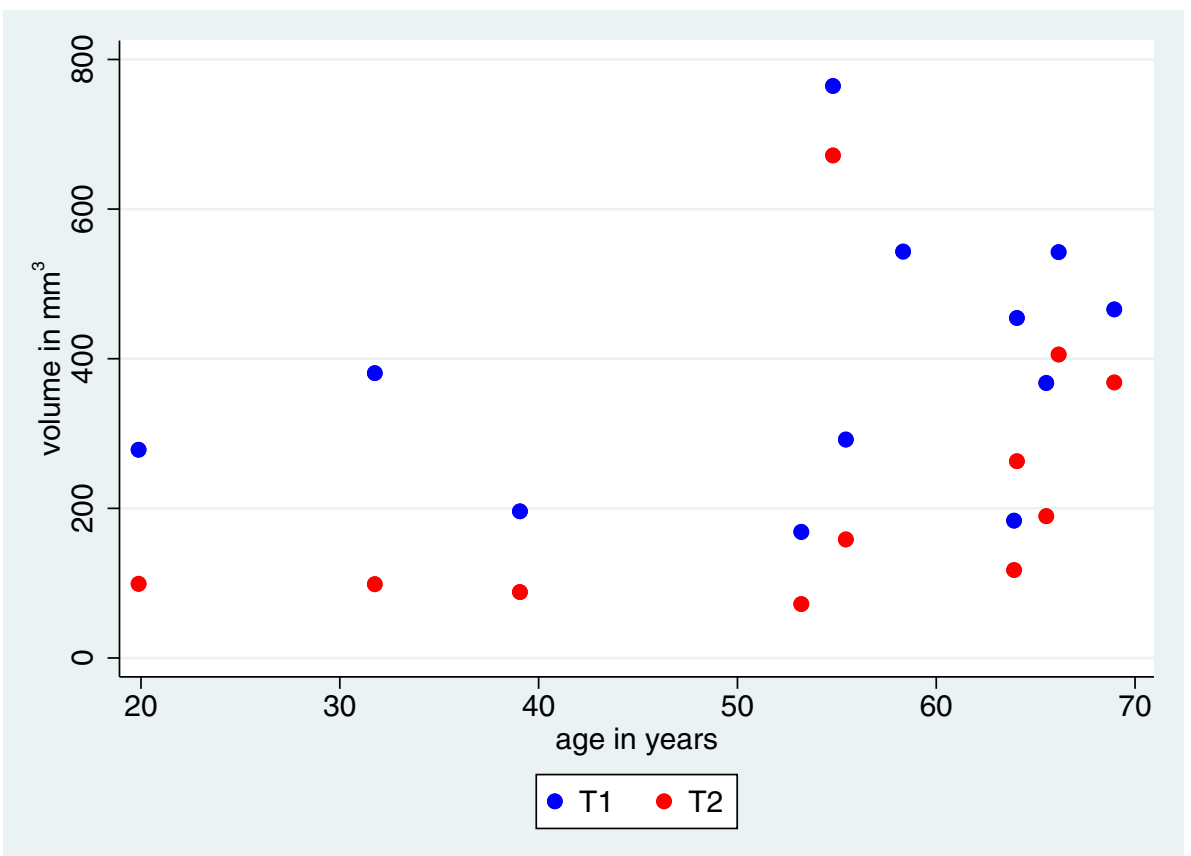

Fig. 5 Relationship between age and bone volume at different time points $\mathrm{T} 1$ and $\mathrm{T} 2$ after augmentation

Spin-Neto et al. introduced the measurement of grafted and residual bone in each cross section through onlay bone grafts and documented bone resorption over the complete bone volume in each grafted area [46].

Lee and Kim obtained the volume of onlay grafts by assigning a range of gray values derived from the donor site of the mandible to grafted bone. However, no reference was found in their work regarding the accuracy of the selected volume and the consistency of standard gray values for bone grafts after transplantation and over the healing period [38].

Kloss et al. measured single site defects on CBCT scans in their height, width, and depth at the cervical level, the middle height of the defect, and at the apical level. All measurements were made on parasagittal sections perpendicular to the longitudinal axis of the adjacent teeth. Based on the radiographic measurements, the graft volume was inferred as the sum of the volumes of two superimposed four-sided rectangular frustums of pyramids [47].

When evaluating edentulous sites, precise identification of stable corresponding landmarks and defined borders within consecutive (CB-)CT scans is crucial for reliable assessment of bone changes at the augmentation site, especially as bone remodeling induces contour changes of the grafted bone block.

This study is based on automated image registration and geometric alignment of consecutive datasets for a more consistent and precise evaluation. As large variability of quantitative gray scale values (GSV) may occur in $\mathrm{CBCT}$ images due to various reasons (e.g., radiation dose, field size, scattered radiation, and limitations of applied reconstruction algorithms), the use of Hounsfield units for tissue characterization in CT may not be applied for CBCT [48]. As the use of quantitative GSV remains essential and cannot be avoided, our study was at least based on CBCT images acquired with identical exposure time and field-of-view to reduce GSV variability.

Furthermore, automated image registration and alignment of all images of the same patient allowed definition of identical ROI prior to evaluation of bone grafts in images of different time points. The residual bone was assessed before bone grafting using stable landmarks outside the ROI to obtain baseline values. Consecutive measurements of bone within the previously marked volume were subtracted by baseline values to obtain the actual volume of bone grafts over time.

Regarding the stability of onlay grafts after 12 months, so far, there is only a limited amount of studies available and data are controversial.

Sbordone et al. detected volume resorption between 35 and $51 \%$ in iliac crest transplants and $45 \%$ for onlay grafts from the chin [45]. In contrast, Kloss et al. stated on mandibular bone grafts in single tooth defects a shrinkage rate of $12.5 \% \pm 7.8 \%$ after 12 months. This is in opposite to our present findings.

On the other hand, Lee and Kim showed a mean graft resorption of $25.4 \%$ at 5.5 months after coverage of onlay grafts from the ascending ramus of the mandible with particulate cortical bone mixed with fibrin sealant and a resorbable collagen membrane $[36,38]$. 
The sparse and highly variable data documented for onlay bone grafts especially from the ascending ramus of the mandible do not allow to predict volume resorption during healing periods of three to six months before the placement of dental implants.

This pilot study showed a continuous three-dimensional bone resorption of autogenous onlay grafts of $43.3 \%$ at 12 months after bone augmentation, using a precise threedimensional assessment based on automated image registration procedure. By adopting identical bony contours and anatomical borders within consecutive CBCT scans, potential manual measurement errors could be reduced.

Further studies with a clearly higher number of patients are necessary to document bone resorption after onlay grafting to establish standard values for clinical recommendations.

\section{Conclusions}

CBCT imaging at different time points combined with an automated image registration procedure allowed to evaluate volume alterations of onlay graft augmentation over a period of 12 months, demonstrating in this study extensive volumetric changes of onlay bone grafts taken from the ascending ramus of the mandible.

Graft resorption and continuous bony remodeling of the grafted site before and after implant insertion have to be carefully considered by the clinician.

Randomized controlled studies with a larger study sample are needed to verify the present findings.

\section{Supplementary Information}

The online version contains supplementary material available at https://doi. org/10.1186/s40729-020-00285-9.

\section{Additional file 1 .}

\section{Acknowledgements}

The authors wish to thank Johanna Greiner and Felicitas Magg for their support during the study.

\section{Authors' contributions}

A.S. and J.F. conceived of the study. A.S., R.J., F.M., J.F. and K.V. performed the analyses. A.S. and T.F. wrote manuscript. The authors read and approved the final manuscript.

\section{Funding}

The work was supported by the Department of Oral and Maxillofacial Surgery, Medical Center - University of Freiburg, Germany, and the Faculty of Medicine, University of Freiburg, Germany.

\section{Availability of data and materials}

Please find all statistical data in the excel sheet enclosed in the supplemental material.

\section{Ethics approval and consent to participate}

All procedures performed in studies involving human participants were in accordance with the ethical standards of the institutional and national research committee and with the 1964 Helsinki declaration and its later amendments or comparable ethical standards. Study approval was obtained from the Ethical Committee of the University Medical Center Freiburg, Germany (138/14).

\section{Consent for publication}

Informed consent was obtained from all individual participants included in the study.

\section{Competing interests}

Andres Stricker declares that he has no conflict of interest. Reinhilde Jacobs declares that he has no conflict of interest. Frederik Maes declares that he has no conflict of interest. Tabea Fluegge declares that he has no conflict of interest. Kirstin Vach declares that she has no conflict of interest. Jonathan

Fleiner declares that he has no conflict of interest.

\section{Author details}

'Center of Implantology, Periodontology and 3D Head-and-Neck Imaging, Konstanz, Germany. ${ }^{2}$ Department of Oral and Maxillofacial Surgery, Medical Center, University of Freiburg, Freiburg, Germany. ${ }^{3}$ Faculty of Medicine, University of Freiburg, Freiburg, Germany. ${ }^{4}$ OMFS-IMPATH Research Group, Department of Imaging and Pathology, Faculty of Medicine, University of Leuven, Leuven, Belgium. ${ }^{5}$ Department of Oral and Maxillofacial Surgery, University Hospitals Leuven, Leuven, Belgium. ${ }^{6}$ Department of Dental Medicine, Karolinska Institutet, Stockholm, Sweden. ${ }^{7}$ ESAT/PSI \& Medical Imaging Research Center, Faculty of Engineering Sciences, University of Leuven, Leuven, Belgium. ${ }^{8}$ Department of Oral and Maxillofacial Surgery, Charité University of Medicine Berlin, Campus Benjamin Franklin, Berlin, Germany. ${ }^{9}$ Institute of Medical Biometry and Statistics, Faculty of Medicine and Medical Center, University of Freiburg, Freiburg, Germany.

Received: 12 July 2020 Accepted: 28 December 2020

Published online: 21 January 2021

\section{References}

1. Cordaro L, Sarzi Amadé D, Cordaro M. Clinical results of alveolar ridge augmentation with mandibular block bone grafts in partially edentulous patients prior to implant placement. Clin Oral Implants Res. 2002:13:103-11.

2. Duttenhoefer F, Nack C, Doll C, Raguse J, Hell B, Stricker A, et al. Long-term peri-implant bone level changes of non-vascularized fibula bone grafted edentulous patients. J Craniomaxillofac Surg. 2015;43:611-5.

3. Fretwurst T, Nack C, Al-Ghrairi M, Raguse J, Stricker A, Schmelzeisen R, et al. Long-term retrospective evaluation of the peri-implant bone level in onlay grafted patients with iliac bone from the anterior superior iliac crest. J Craniomaxillofac Surg. 2015:43:956-60.

4. Chappuis V, Engel O, Reyes M, Shahim K, Nolte L, Buser D. Ridge alterations post-extraction in the esthetic zone: a 3D analysis with CBCT. J Dent Res. 2013;92(12_suppl):195S-201S. https://doi.org/10.1177/0022034513506713.

5. Chappuis V, Cavusoglu Y, Buser D, Arx T. Lateral ridge augmentation using autogenous block grafts and guided bone regeneration: a 10-year prospective case series. Study Clin Implant Dent Related Res. 2016;07. https://doi.org/10.1111/cid.12438.

6. Braut $V$, Bornstein MM, Belser $U$, Buser D. Thickness of the anterior maxillary facial bone wall-a retrospective radiographic study using cone beam computed tomography. Int J Periodont Restorative Dentistry. 2011:31:125-31

7. Chiapasco M, Zaniboni M, Boisco M. Augmentation procedures for the rehabilitation of deficient edentulous ridges with oral implants. Clin Oral Implants Res. 2006;17 Suppl 2(10):136-59.

8. Buser D, Chappuis V, Kuchler U, Bornstein M, Wittneben J, Buser R, et al. Long-term stability of early implant placement with contour augmentation. J Dent Res. 2013;92(12 Suppl):176S-82S.

9. Donos N, Mardas N, Chadha V. Clinical outcomes of implants following lateral bone augmentation: systematic assessment of available options (barrier membranes, bone grafts, split osteotomy). J Clin Periodontol. 2008; 35(8 Suppl):173-202

10. Chiapasco M, Casentini P, Zaniboni M. Bone augmentation procedures in implant dentistry. Int J Oral Maxillofac Implants. 2009;24(Suppl):237-59.

11. Burchardt H. The biology of bone graft repair. Clin Orthop Relat Res. 1983; 174:28-42.

12. Boyne $P$, James R. Grafting of the maxillary sinus floor with autogenous marrow and bone. J Oral Surg (American Dental Association: 1965). 1980;38:613-6. 
13. Wood R, Moore D. Grafting of the maxillary sinus with intraorally harvested autogenous bone prior to implant placement. Int J Oral Maxillofac Implants. 1988;3:209-14

14. Hunt $\mathrm{D}$, Jovanovic $\mathrm{S}$. Autogenous bone harvesting: a chin graft technique for particulate and monocortical bone blocks. Int J Periodontics Restorative Dent. 1999:19:165-73.

15. Schliephake H, Dard M, Planck H, Hierlemann H, Jakob A. Guided bone regeneration around endosseous implants using a resorbable membrane vs a PTFE membrane. Clin Oral Implants Res. 2000;11:230-41.

16. Hirsch J, Ericsson J. Maxillary sinus augmentation using mandibular bone grafts and simultaneous installation of implants. A surgical technique. Clin Oral Implants Res. 1991;2:91-6.

17. Isaksson S, Alberius P. Maxillary alveolar ridge augmentation with onlay bone-grafts and immediate endosseous implants. J Craniomaxillofac Surg. 1992;20:2-7.

18. Tolman D. Reconstructive procedures with endosseous implants in grafted bone: a review of the literature. Int J Oral Maxillofac Implants. 1995;10:275-94.

19. Rasmusson L, Meredith N, Kahnberg K, Sennerby L. Stability assessments and histology of titanium implants placed simultaneously with autogenous onlay bone in the rabbit tibia. Int J Oral Maxillofac Surg. 1998;27:229-35.

20. Johansson B, Grepe A, Wannfors K, Aberg P, Hirsch J. Volumetry of simulated bone grafts in the edentulous maxilla by computed tomography: an experimental study. Dentomaxillofac Radiol. 2001;30:153-6.

21. Sjöström M, Sennerby L, Nilson H, Lundgren L. Reconstruction of the atrophic edentulous maxilla with free iliac crest grafts and implants: a 3-year report of a prospective clinical study. Clin Implant Dent Relat Res. 2007;9: 46-59.

22. Nyström E, Ahlqvist J, Gunne J, Kahnberg K. 10-year follow-up of onlay bone grafts and implants in severely resorbed maxillae. Int J Oral Maxillofac Surg. 2004;33:258-62.

23. Wiltfang J, Jätschmann N, Hedderich J, Neukam F, Schlegel K, Gierloff M. Effect of deproteinized bovine bone matrix coverage on the resorption of iliac cortico-spongeous bone grafts - a prospective study of two cohorts. Clin Oral Implants Res. 2014;25:e127-32.

24. De Bruyn H, Bouvry P, Collaert B, De Clercq C, Persson G, Cosyn J. Longterm clinical, microbiological, and radiographic outcomes of BrånemarkTM implants installed in augmented maxillary bone for fixed full-arch rehabilitation. Clin Implant Dent Relat Res. 2013;15:73-82.

25. Maiorana C, Sigurtà D, Mirandola A, Garlini G, Santoro F. Bone resorption around dental implants placed in grafted sinuses: clinical and radiologic follow-up after up to 4 years. Int J Oral Maxillofac Implants. 2005;20:261-6.

26. Malchiodi L, Quaranta A, D'Addona A, Scarano A, Quaranta M. Jaw reconstruction with grafted autologous bone: early insertion of osseointegrated implants and early prosthetic loading. J Oral Maxillofacial Surg. 2006;64:1190-8.

27. Proussaefs P. Clinical and histologic evaluation of the use of mandibular tori as donor site for mandibular block autografts: report of three cases. Int J Periodontics Restorative Dent. 2006;26:43-51.

28. Arx T, Buser D. Horizontal ridge augmentation using autogenous block grafts and the guided bone regeneration technique with collagen membranes: a clinical study with 42 patients. Clin Oral Implants Res. 2006; 17:359-66.

29. Fredholm U, Bolin A, Andersson L. Preimplant radiographic assessment of available maxillary bone support. Comparison of tomographic and panoramic technique. Swed Dent J. 1993;17:103-9.

30. Bolin A, Eliasson S, Beetzen M, Jansson L. Radiographic evaluation of mandibular posterior implant sites: correlation between panoramic and tomographic determinations. Clin Oral Implants Res. 1996;7:354-9.

31. Dasmah A, Thor A, Ekestubbe A, Sennerby L, Rasmusson L. Particulate vs. block bone grafts: three-dimensional changes in graft volume after reconstruction of the atrophic maxilla, a 2-year radiographic follow-up. J Craniomaxillofac Surg. 2012;40:654-9.

32. Bornstein M, Horner K, Jacobs R. Use of cone beam computed tomography in implant dentistry: current concepts, indications and limitations for clinical practice and research. Periodontol. 2017;73:51-72.

33. Degidi M, Nardi D, Piattelli A. 10-year follow-up of immediately loaded implants with TiUnite porous anodized surface. Clin Implant Dent Relat Res. 2012;14:828-38.

34. Schropp L, Wenzel A, Stavropoulos A. Early, delayed, or late single implant placement: 10-year results from a randomized controlled clinical trial. Clin Oral Implants Res. 2014;25:1359-65.
35. Temmerman A, Keestra JA, Coucke W, Teughels W, Quirynen M. The outcome of oral implants placed in bone with limited bucco-oral dimensions: a 3-year follow-up study. J Clin Periodontol. 2015;42:311-8.

36. Spin-Neto R, Stavropoulos A, Violin Dias Pereira L, Marcantonio E, Wenzel A. Fate of autologous and fresh-frozen allogeneic block bone grafts used for ridge augmentation. A CBCT-based analysis. Clin Oral Implants Res. 2013;24: 167-73.

37. Anitua E, Murias-Freijo A, Alkhraisat MH. Implant site under-preparation to compensate the remodeling of an autologous bone block graft. J Craniofac Surg. 2015;26:e374-7

38. Lee HG, Kim YD. Volumetric stability of autogenous bone graft with mandibular body bone: cone-beam computed tomography and threedimensional reconstruction analysis. J Korean Assoc Oral Maxillofac Surg. 2015;41:232-9.

39. Monje A, Monje F, Hernández-Alfaro F, Gonzalez-García R, Suárez-López del Amo F, Galindo-Moreno P, et al. Horizontal bone augmentation using autogenous block grafts and particulate xenograft in the severe atrophic maxillary anterior ridges: a cone-beam computerized tomography case series. J Oral Implantol. 2015;41:366-71.

40. Maes F, Collignon A, Vandermeulen D, Marchal G, Suetens P. Multimodality image registration by maximization of mutual information. IEEE Trans Med Imaging. 1997;16:187-98.

41. Aghaloo TL, Moy PK. Which hard tissue augmentation techniques are the most successful in furnishing bony support for implant placement? Int J Oral Maxillofacial Implants. 2007;22:49-70.

42. Nyström E, Legrell P, Forssell A, Kahnberg K. Combined use of bone grafts and implants in the severely resorbed maxilla. Postoperative evaluation by computed tomography. Int J Oral Maxillofac Surg. 1995;24:20-5.

43. Sjöström M, Sennerby L, Lundgren L. Bone graft healing in reconstruction of maxillary atrophy. Clin Implant Dent Relat Res. 2013;15:367-79.

44. Sbordone C, Toti P, Martuscelli R, Guidetti F, Sbordone L, Ramaglia L. A 5year implant follow-up in maxillary and mandibular horizontal osseous onlay grafts and native bone. J Oral Implantol. 2015;41:570-8.

45. Sbordone L, Toti P, Menchini-Fabris G, Sbordone C, Piombino P, Guidetti F. Volume changes of autogenous bone grafts after alveolar ridge augmentation of atrophic maxillae and mandibles. Int J Oral Maxillofac Surg. 2009;38:1059-65.

46. Spin-Neto R, Stavropoulos A, Coletti F, Pereira L, Marcantonio E, Wenzel A. Remodeling of cortical and corticocancellous fresh-frozen allogeneic block bone grafts--a radiographic and histomorphometric comparison to autologous bone grafts. Clin Oral Implants Res. 2015;26:747-52.

47. Kloss FR, Offermanns V, Kloss-Brandstätter A. CE. Comparison of allogeneic and autogenous bone grafts for augmentation of alveolar ridge defects-a 12-month retrospective radiographic evaluation. Clin Oral Implants Res. 2018;29:1163-75.

48. Pauwels R, Jacobs R, Singer SR, Mupparapu M. CBCT-based bone quality assessment: are Hounsfield units applicable? Dentomaxillofacial Radiol. 2015; 44:20140238. https://doi.org/10.1259/dmfr.20140238.

\section{Publisher's Note}

Springer Nature remains neutral with regard to jurisdictional claims in published maps and institutional affiliations.

\section{Submit your manuscript to a SpringerOpen ${ }^{\circ}$ journal and benefit from:}

- Convenient online submission

- Rigorous peer review

- Open access: articles freely available online

High visibility within the field

- Retaining the copyright to your article

Submit your next manuscript at $>$ springeropen.com 